\title{
Surgical Repair Outcomes of Inflammatory and Degenerative Abdominal Aortic Aneurysms
}

\author{
Javad Salimi ${ }^{1}$, Zahra Omrani ${ }^{2}$ and Roozbeh Cheraghali ${ }^{3}$ \\ ${ }^{1}$ Department of Vascular Surgery, Tehran University of Medical Sciences, Tehran, Iran \\ ${ }^{2}$ Department of Surgery, Iran University of Medical Sciences, Tehran, Iran \\ ${ }^{3}$ Department of Vascular Surgery, Golestan University of Medical Sciences, Gorgan, Iran
}

\begin{abstract}
Inflammatory abdominal aortic aneurysm (IAAA) is characterised by thick aortic wall, with perianeurysmal fibrosis extending to adjacent organs. This study aimed to determine the early surgical outcomes of 10 IAAAs and compare the risk factors, perioperative complications, and 30-day mortality of these patients to 50 degenerative AAAs (dAAA), who underwent open repair in Sina Hospital, Tehran, Iran. The study was conducted from 2011 to 2019. IAAA patients were followed for $22.7 \pm 3.8$ months. The frequency of IAAA was $4.9 \%$. All IAAA patients were males and smokers. There was no statistical difference in preoperative risk factors (age, smoking, hypertension, chronic kidney disease, ischemic heart disease, chronic obstructive pulmonary disease), operation time, blood loss, postoperative complications between dAAA and IAAA patients. The thirty-day mortality rate of open surgeries in degenerative and inflammatory cases was $8 \%(N=4)$ and $10 \%(N=1)$, respectively. Open repair with proxi$\mathrm{mal} / \mathrm{and} / \mathrm{or}$ distal clamping, avoids severe adhesiolysis, procedure-related morbidity, and 30-day perioperative mortality.
\end{abstract}

Key Words: Inflammatory abdominal aortic aneurysm, Open surgery, Mortality, Outcome, Degenerative aortic aneurysm.

How to cite this article: Salimi J, Omrani Z, Cheraghali R. Surgical Repair Outcomes of Inflammatory and Degenerative Abdominal Aortic Aneurysms. J Coll Physicians Surg Pak 2022; 32(03):377-379.

\section{INTRODUCTION}

Inflammatory abdominal aortic aneurysms (IAAA) are characterised by the thickness of the aortic wall, with perianeurysmal fibrosis, expanding to adjacent organs. Duodenum, left renal vein, and ureters are mostly involved. ${ }^{1}$ They can be asymptomatic, but when symptoms occur, patients present with abdominal and/or back pain, fatigue, and weight loss. Erythrocyte sedimentation rate may be elevated in this group of patients. IAAA differences with a mycotic aneurysm can be distinguished by the absence of infection. Ureters fibrous encasement leads to obstruction, hydronephrosis and renal failure. $^{2}$ Open surgical repair (OSR) and endovascular aneurysm repair (EVAR) are two treatment methods for IAAA. ${ }^{1}$ Periaortic fibrosis (PAF) makes a hostile operative abdomen; and adhesions may involve the duodenum, the left renal vein, and other structures, which can lead to iatrogenic injury. Thus, it may lead to higher intra and postoperative morbidity and mortality of IAAA.

Correspondence to: Roozbeh Cheraghali, Department of

Vascular Surgery, Golestan University of Medical

Sciences, Gorgan, Iran

E-mail: roozbehcheraghali81@gmail.com

Received: March 28, 2021; Revised: September 16, 2021;

Accepted: October 15, 2021

DOI: https://doi.org/10.29271/jcpsp.2022.03.377
Development of visceral organ complications in previously involved or uninvolved structures, attributable to ongoing fibrosis after surgical intervention, are well-known complications; but the impact of ongoing inflammation on long-term survival remains to be determined. ${ }^{3,4}$

The aim of this series was to determine the surgical repair outcomes (postoperative complications and 30-day mortality) of inflammatory and degenerative abdominal aortic aneurysms.

\section{METHODOLOGY}

This series consisted of 10 elective IAAA patients, who underwent open surgery at Sina Hospital, Tehran, from September 2011 to December 2019. Emergency ruptured aneurysm repair, suprarenal and thoracic aneurysms, defects in data, and inability to track the patient via telephone contact were excluded. Fifty dAAA patients, who electively were treated by open repair during the study period, were also included. All patients signed the consent form. IRB approval was obtained from Tehran University of Medical Sciences; REC1399.419. A checklist of required data (age, gender, comorbidities, smoking, aneurysm diameter, ESR, $\mathrm{CRP}$, operation time, blood loss during surgery, prolonged paralytic ileus, creatinine rise, and deaths in 30 days) was prepared and used to extract data from patients' medical case files. Computed tomography (CT) was used for the diagnosis of AAA and differentiating between inflammatory and degenerative types. All IAAAs were infrarenal; and iliac arteries were not involved in any of them. Histopathologic examinations confirmed aneurysms to be inflammatory. SPSS Version 18 soft- 
ware was used to analyse the data. In the descriptive analysis, the frequency, percentage, mean, and standard deviation were reported.

Following midline incision, the neck of the aneurysm was dissected and released near renal artery origins, and then near right and left iliac arteries. In some cases, left renal vein was ligated to find more secure proximal arterial control, if applicable. Ureters were explored and distal control was taken. Vascular clamps were used to get proximal and distal control and aneurysms were opened without releasing the adhesions over the sac. A tube graft of the aorta was created in all 10 IAAAs (Figure 1).



Figure 1: Thickinflammatory aortic aneurysm sac (A: CTscan; B: Surgery).

Table I: Comparison of 30-day mortality and risk factors of IAAA and dAAA patients. (Non-significant: pvalue $>0.05$ ).

\begin{tabular}{|c|c|c|c|}
\hline & IAAA $^{1}(n=10)$ & dAAA $^{2}(n=50)$ & p-value \\
\hline Age & $68.1 \pm 5.6$ & $68.6 \pm 8.6$ & $\begin{array}{l}0.814 \\
\text { (NS) }\end{array}$ \\
\hline Gender (male) & $10(100 \%)$ & $48(96 \%)$ & $>0.999$ \\
\hline Hypertension & $5(50 \%)$ & $26(52 \%)$ & $>0.999$ \\
\hline $\begin{array}{l}\text { Ischemic heart } \\
\text { disease }\end{array}$ & $2(20 \%)$ & $19(38 \%)$ & 0.470 \\
\hline $\begin{array}{l}\text { Chronic Kidney } \\
\text { disease }\end{array}$ & $1(10 \%)$ & $6(12 \%)$ & $>0.999$ \\
\hline COPD & $2(20 \%)$ & $3(6 \%)$ & 0.190 \\
\hline CVA & $1(10 \%)$ & $3(6 \%)$ & 0.528 \\
\hline Smoking & $10(100 \%)$ & $45(90 \%)$ & 0.578 \\
\hline $\begin{array}{l}\text { Aneurysm } \\
\text { diameter }(\mathrm{cm})\end{array}$ & $74.4 \pm 9.8$ & $72.6 \pm 14.0$ & 0.723 \\
\hline 30 day mortality & $1(10 \%)$ & $4(8 \%)$ & $>0.999$ \\
\hline $\begin{array}{l}\text { Follow up period } \\
\text { (months) }\end{array}$ & $22.7 \pm 3.8$ & $23.8 \pm 5.1$ & 0.518 \\
\hline $\begin{array}{l}\text { Operation time } \\
\text { (min) }\end{array}$ & $140.0 \pm 25.4$ & $132.7 \pm 33.0$ & 0.517 \\
\hline Blood loss (ml) & $634.0 \pm 119.6$ & $869.0 \pm 575.1$ & 0.206 \\
\hline $\begin{array}{l}\text { Hospital stay } \\
\text { (days) }\end{array}$ & $6.9 \pm 2.3$ & $7.2 \pm 2.8$ & 0.758 \\
\hline $\begin{array}{l}\text { Creatinine rise } \\
(\mathrm{mg} / \mathrm{dl})\end{array}$ & $1(10 \%)$ & $7(14 \%)$ & $>.999$ \\
\hline Prolonged ileus & $1(10 \%)$ & $10(20 \%)$ & 0.671 \\
\hline
\end{tabular}

\section{RESULTS}

During the study period, the frequency of IAAA was $4.9 \%$. Patients were evaluated in two groups: 50 dAAA (open repair) and 10 Inflammatory AAAs. All IAAA patients were males and smokers. Characteristics of each group are summarised in Table I. All of the IAAAs had elevated ESR and CRP. The aneurysm diameter of IAAAs was $74.4 \pm 9.8$ (60-84) $\mathrm{mm}$. They were admitted when they were symptomatic and required urgent intervention.

In the IAAAs group, one patient died 2 weeks after the operation due to respiratory failure. He had severe COPD for 10 years and could not be weaned off the mechanical ventilator. A transient rise of creatinine was noted in one patient (10\%); and he was discharged after 10 days with creatinine in the normal range. Prolonged paralytic ileus also occurred in one of the IAAA group (10\%).

The mean operation time in degenerative and inflammatory patients was $132.76 \pm 33.08$ and $140.00 \pm 25.49$ minutes, respectively (Table I). The mean follow-up time of IAAAs was $22.7 \pm 3.8$ months. There was no statistical difference between the frequency of smokers in dAAA and IAAA patients.

\section{DISCUSSION}

IAAA was first described by Walker et al. in 1972. ${ }^{5}$ It occurs in 3\% to $10 \%$ of all AAA, which is more common in men. It is highly associated with smoking, and younger presentation age. Moreover, it has a lower risk of rupture than non-inflammatory AAA. ${ }^{1-4}$ In this study, the frequency of IAAA was $4.9 \%$. Chief complaints in all IAAA patients were abdominal and low back pain, but fever was not detected in this group. In susceptible patients, a severe form of inflammatory changes, secondary to atherosclerotic plaque antigens, may cause inflammatory aneurysms. ${ }^{6,7}$

lino et $a l^{8}{ }^{8}$ showed $90 \%$ sensitivity for computed tomography. Computerised tomography is the most reliable diagnostic modality for IAAA. CT scan and MR angiography are helpful for diagnosis and follow-up. ${ }^{8}$ In this series, patients were followed for $23.8 \pm 5.1$ months, but CT scan was not used routinely during the follow-up period.

Control of hypertension, hyperlipidemia, smoking cession are important for medical management. ${ }^{1}$ Most authors agree that corticosteroids do not alter the long-term development of inflammatory aneurysms and that operative repair of the aneurysm is the treatment of choice. ${ }^{9}$ Although surgical intervention appears prudent once the diameter of the aneurysm exceeds $5.5 \mathrm{~cm}$. The endovascular repair can be done, if it is anatomically feasible with a lower rate of inflammatory response resolution. The surgical challenge of IAAA lies in the difficulty of the dissection and control of the proximal and distal aorta, which leads to a three-times-higher perioperative morbidity and mortality rate $(7.9 \%$ vs. $2.4 \%) .{ }^{9}$ Nuellari et al. declared the fibrotic process complicated surgical dissection and exposure of the aorta. However, it did not seem to influence the results of surgery in terms of survival. Surgical injury to the 
adherent organs has been reported in $4.5 \%$ to $15 \%$ of cases. ${ }^{3}$ No controlled clinical trials have evaluated the long-term efficacy of steroids for IAAAs. ${ }^{4}$ In this study, IAAAs were symptomatic, and surgery was conducted within one or two days of admission, so corticosteroids were not prescribed preoperatively.

This surgical technique in patients suffering from IAAA avoids retroperitoneal approach and extensive adhesiolysis. In a single-institution study of 18 IAAA patients, who were followed for a mean of 61 months, IAAA repair was associated with a higher incidence of anastomotic aneurysms than dAAA repair. ${ }^{10}$ Although in Bonati et al., early outcomes and late cumulative survival rates were not significantly different. ${ }^{11}$ In this study, the mean follow-up period was 23 months and anastomotic pseudoaneurysm was not detected in the IAAA group, so larger or multicentre studies are needed to detect probable anastomotic pseudoaneurysm.

Finally, in IAAAs, medical therapy is sometimes mandatory postoperatively to reduce the inflammation and accompanying fibrosis that usually involves adjacent organs. For example, some case series employed corticosteroids and immunosuppressive agents, such as methotrexate, cyclophosphamide, and azathioprine. ${ }^{12}$

\section{CONCLUSION}

The results of this research suggest open repair with proxi$\mathrm{mal} / \mathrm{and} /$ or distal clamping in patients with IAAAs avoids severe adhesiolysis, can reduce procedure-related morbidity, and 30day perioperative mortality.

\section{ETHICALAPPROVAL:}

This study was approved in TUMS Ethics Committee.

\section{PATIENTS' CONSENT:}

Consent forms were obtained from all patients. Consent for publication was obtained.

DATA AVAILABILITY: Electronic database is available at Sina Hospital of Tehran University of Medical Sciences.

\section{CONFLICT OF INTEREST:}

The authors declared no conflict of interest.

\section{AUTHORS' CONTRIBUTION:}

JS, ZO, RC: Data collection, and analysis.

ZO: Investigation.

JS, ZO, RC: Writing the manuscript.

All authors approved the final version for publication and are accountable for all aspect of the work.

\section{REFERENCES}

1. Stone WM, Fankhauser GT. Inflammatory aneurysms treated with EVAR. Semin Vasc Surg 2012; 25(4):227-31. doi: 10.1053/j.semvascsurg.2012.09.008.

2. Sterpetti AV, Hunter WJ, Feldhaus RJ, Chasan P, McNamara $\mathrm{M}$, Cisternino $\mathrm{S}$, et al. Inflammatory aneurysms of the abdominal aorta: Incidence, pathologic, and etiologic considerations. J Vasc Surg 1989; 9(5):643-9. doi: 10.1067/ mva.1989.vs0090643.

3. Nuellari E, Prifti E, Esposito G, Kapedani E. Surgical treatment of inflammatory abdominal aortic aneurysms: Outcome and predictors analysis. Med Arch 2014; 68(4): 244-8. doi: 10.5455/medarh.2014.68.244-248.

4. Vaglio A, Buzio C. Chronic periaortitis: A spectrum of diseases. Curr Opin Rheumatol 2005; 17(1):34-40. doi: 10.1097/01.bor.0000145517.83972.40.

5. Walker DI, Bloor K, Williams G, Gillie I. Inflammatory aneurysms of the abdominal aorta. Br J Surg 1972; 59(8):609-14. doi: 10.1002/bjs.1800590807.

6. Mitchinson MJ. Chronic periaortitis and periarteritis. Histopathology 1984; 8(4):589-600. doi: 10.1111/j.13652559.1984.tb02371.x.

7. Parums DV, Brown DL, Mitchinson MJ. Serum antibodies to oxidized low density lipoprotein and ceroid in chronic periaortitis. Arch Pathol Lab Med 1990; 114(4):383-7.

8. Iino $M$, Kuribayashi S, Imakita S, Takamiya $M$, Matsuo $H$, Ookita $Y$, et al. Sensitivity and specificity of CT in the diagnosis of inflammatory abdominal aortic aneurysms. J Comput Assist Tomogr 2002; 26(6):1006-12. doi: 10.1097/ 00004728-200211000-00026.

9. Hellmann DB, Grand DJ, Freischlag JA. Inflammatory abdominal aortic aneurysm. JAMA 2007; 297(4):395-400. doi: 10.1001/jama.297.4.395.

10. Paravastu SC, Ghosh J, Murray D, Farquharson FG, Serracino-Inglott F, Walker MG. A systematic review of open versus endovascular repair of inflammatory abdominal aortic aneurysms. Eur J Vasc Endovasc Surg 2009; 38(3):291-7. doi: 10.1016/j.ejvs.2009.05.005.

11. Bonati L, Rubini P, Japichino GG, Parolari A, Contini S, Zinicola $\mathrm{R}$, et al. Long-term outcome after inflammatory abdominal aortic aneurysm repair: Case-matched study. World J Surg 2003; 27(5):539-44. doi: 10.1007/s00268003-6706-4.

12. Capoccia L, Riambau V. Endovascular repair versus open repair for inflammatory abdominal aortic aneurysms. Cochrane Database of Syst Rev 2015; 4:CD010313. doi: 10.1002/14651858.CD010313.pub2. 